\title{
EL SUICIDIO DESDE UN ENFOQUE PSICOSOCIAL Y DE SALUD COMUNITARIA: LOS RESULTADOS DEL DIAGNÓSTICO EN SANTA MARÍA DE DOTA, COSTA RICA
}

\author{
SUICIDE FROM A PSYCHOSOCIAL AND COMMUNITY HEALTH \\ APPROACH: THE RESULTS OF THE DIAGNOSIS \\ IN SANTA MARIA DE DOTA, COSTA RICA
}

\section{Carolina Castillo Echeverría Adriana Maroto Vargas}

Recibido: 02/05/2017 Aceptado: 14/07/2016

\section{Resumen}

El presente artículo expone los resultados del diagnóstico comunitario que se realizó en el marco del proyecto de la Universidad de Costa Rica, titulado "Desarrollo de capacidades en la comunidad de Santa María de Dota para la prevención del suicidio"; el cual tenía como objetivo contribuir a la comprensión de este fenómeno en dicha comunidad a partir de la identificación de aquellos factores que inciden sobre la conducta suicida. Se utilizó una metodología cualitativa y participativa con técnicas como entrevistas, grupos de discusión y talleres. En los resultados se exponen los principales problemas de la comunidad y los aspectos positivos considerados respectivamente como factores de riesgo y factores protectores para el suicidio, así como la percepción que se tiene de este y las herramientas comunitarias para enfrentarlo.

Palabras clave: Suicidio; enfoque psicosocial; salud comunitaria; factores de riesgo; factores protectores.

\begin{abstract}
The present article at hand presents the results of the community assessment that was carried out as part of the project "Capacity building in the community of Santa Maria de Dota for suicide prevention" from the University of Costa Rica. The former aimed to better understand suicide in this community by identifying those factors that influence suicidal behavior. A qualitative and participatory approach, with techniques such as interviews, focus groups, and workshops, was used. The results expose the main problems of the community considered as risk factors for suicide, the positive qualities that are considered as protective factors, and the perception people have of suicide as well as the strategies the community has to face this problem.
\end{abstract}

Keywords: Suicide; psychosocial focus; community health; risk factors; protective factors. 


\section{Introducción}

En el presente artículo se exponen los principales resultados del diagnóstico comunitario que se llevó a cabo durante el 2014, en el marco del proyecto de Acción Social de la Universidad de Costa Rica titulado "Desarrollo de capacidades en la comunidad de Santa María de Dota para la prevención del suicidio"; el cual tenía como objetivo contribuir a la comprensión de dicho fenómeno en esta comunidad al identificar aquellos factores que inciden en la conducta suicida.

La Zona de los Santos, compuesta por los cantones de Dota, Tarrazú y León Cortés, presenta las tasas más altas de intentos de suicidio en el país, según datos el Ministerio de Salud (2014). A pesar de ser una problemática común en toda la zona, el proyecto se enfocó en el cantón de Dota debido a una solicitud que plantearon los líderes de la comunidad a la Rectoría de la Universidad de Costa Rica.

Tradicionalmente, el suicidio ha sido estudiado desde enfoques clínicos e individuales; no obstante, en este caso se consideró que el alcance de dichos enfoques es insuficiente, pues no permite comprender aquellos aspectos sociales y comunitarios que pueden incidir sobre el suicidio en esta localidad en particular. Por tanto, teóricamente se partió de un enfoque psicosocial y de salud comunitaria para su estudio y abordaje.

Así mismo, el diagnóstico comunitario se realizó con técnicas cualitativas participativas, tales como entrevistas, grupos de discusión y talleres, de forma que la problemática se pudiera conocer a través de la perspectiva de sus habitantes. Fue así como se elaboró el diagnóstico.

\section{Contextualización del suicidio}

Mundialmente, el suicidio se encuentra dentro las veinte causas principales de muerte. Alrededor de un millón de personas en el mundo se quitan la vida cada año y los números parecieran ir en aumento, de manera que se ha convertido en un problema de salud pública (Organización Panamericana de la Salud 1).

En América ocurren aproximadamente 65000 muertes por año a causa del suicidio, lo cual equivale a una tasa de 7.3 por ciento por 100000 habitantes de acuerdo con datos que comprenden el periodo 2005-2009 (Organización Panamericana de la Salud 5). Específicamente, en América Central, el Caribe y México la tasa de suicidios fue de 5.06 por cada 100000 habitantes durante ese periodo (Organización Panamericana de la Salud 14). Además, se identificó que los grupos de edad con una mayor tasa de suicidio son los que se encuentran entre los 20 y 24 años, con una tasa de 7.83; de 60 a 69 años con una tasa de 7.64 y las personas de 70 años o más con una tasa de 10.48 (Organización Panamericana de la Salud, 2014). Así mismo, la tasa de suicidios en hombres (8.11) es superior a la de las mujeres (1.97), lo cual resulta congruente con la tendencia mundial (Organización Panamericana de la Salud, 2014). 
En comparación al resto de los países de Centroamérica, Costa Rica posee la segunda tasa más alta de suicidios después de El Salvador, con un promedio de 7,06 por 100000 habitantes durante el periodo 2005-2009 (Organización Panamericana de la Salud, 2014). Según otro estudio que abarcó de 1988 al 2008, en Costa Rica la tasa de suicidios aumentó considerablemente, al pasar de 4.93 en 1988 a 7.14 en el 2008 (Organización Panamericana de la Salud, 2011). Al mismo tiempo, las tasas según género aumentaron tanto en hombres como en mujeres; sin embargo, son los primeros quienes presentan una mayor prevalencia durante todo el periodo, al pasar de una tasa de 8.40 en 1988 a 12.36 en el 2008, mientras que en las mujeres creció de 1.33 en 1988 a 1.74 en 2008 (Organización Panamericana de la Salud, 2011). En cuanto a los grupos etarios, entre el 2006 y el 2008, las personas de 75 a 79 años presentaron las tasas más altas de suicidios (de 7.7 a 13.41). Pese a esto, en el grupo entre los 35 y los 40 años estas también fueron significativas, pues presentaron un aumento considerable pasando de una tasa aproximada de 8.5 en el 2006 a 12 en el 2008 (Organización Panamericana de la Salud, 2011). Estos datos demuestran que el análisis de dicho problema, tanto en la región centroamericana como en Costa Rica, es de gran relevancia en la actualidad.

Si bien se puede apreciar que Costa Rica presenta una tasa más baja en comparación con América Latina, los datos muestran la tendencia al aumento, lo cual hace urgente una investigación que retroalimente la definición de políticas públicas. Arias y Blanco (2010) señalan que más allá de entender el suicido como un problema epidemiológico de tendencia creciente, se debe estudiar el suicidio en comunidades muy particulares, pues este podría tener múltiples y diversos significados y causas, así como diversidad de representaciones culturales. De tal manera que el diagnóstico local se convierte en una herramienta importante.

Respecto al lugar de residencia, según datos del Organismo de Investigación Judicial, para el 2013 la provincia que presentó la mayor tasa de suicidios en Costa Rica fue Guanacaste con un 8.9, seguido de San José con un 8.5 y Limón con una tasa de 8.0 (Organismo de Investigación Judicial, 2013). Así mismo, según datos por cantón de ese mismo año, dos de los tres cantones que conforman la zona de los Santos, ubicados en la provincia de San José, se encuentran en los primeros lugares con mayores tasas de suicidios en el país. Tarrazú se ubica en la cuarta posición con una tasa de 1.68 y Dota, localidad en que se basa esta investigación, se localiza en el undécimo lugar con una tasa de 1.32 (Ministerio de Salud, 2013).

Si bien existen estas tendencias generales, no se puede perder de vista -especialmente desde los abordajes psicosociales- que las tasas de suicidio varían en cada país, región y década, en relación directa con los múltiples factores asociados a la conducta suicida (Sáenz Rojas, 2011). De tal manera, factores de riesgo, tanto sociales (pobreza, desempleo, drogadicción) como psicológicos (depresión, duelo), podrían incidir en el aumento de las cifras en Costa Rica (Chaves Moreno, Madrigal Ramírez, Vargas Sanabria y Abarca Villalobos, 2008). Ante esto, el abordaje psicosocial y comunitario 
del suicidio se torna de gran utilidad para identificar los factores de riesgo que pueden provocar la presencia de conductas suicidas en localidades particulares, así como para formular estrategias de prevención y abordaje desde el mismo espacio comunitario.

\section{Aspectos teóricos}

\section{El enfoque psicosocial del suicidio}

De acuerdo con Carmona Parra, Torbón Hoyos, Jaramillo Estrada y Areiza Sánchez (2010), el suicidio debe analizarse desde una perspectiva interaccionista, ya que puede ser una expresión de un acto individual gestado en la dinámica de esa persona con el mundo que le rodea, en donde pueden intervenir factores sociales en la construcción y significación del acto.

Según la teoría de las representaciones sociales, en el proceso de interacción con otras personas se comparten los universos simbólicos que le otorgan sentido a la realidad y que dirigen el accionar de las personas (Moscovici, 1979). Por tanto, la forma de entender el suicidio depende de cómo, en un contexto social y cultural particular, se construye y reconstruye el acto continuamente en un proceso de intercomunicación individual-social (García Alba et al., 2011). Desde este punto de vista, se debe tratar de identificar el papel de las instituciones y los grupos de pertenencia del individuo, ya que pueden influir en los significados que se le den al suicidio, ya sea para estimularlo o desincentivarlo:

Esta perspectiva interaccionista permite comprender los intentos de suicidios y los suicidios en niños y adolescentes, como actos socialmente construidos, en los cuales los factores biológicos, psicológicos y sociológicos tienen un lugar, pero no en calidad de determinismos mecánicos, o variables independientes que explicarían el fenómeno como una resultante o variable dependiente, sino como aspectos del mundo físico y simbólico con los que los niños y adolescentes se relacionan de una manera activa, alterándolos, transformándolos, interpretándolos, resignificándolos, recreándolos e incluso reinventándolos (Carmona Parra et al. 94-95).

Asimismo, el suicidio no se puede explicar a partir de una única variable, ni se pueden generalizar sus causas a todos los diferentes casos, pues cada uno responderá a la conjunción de distintos factores. Por tanto, para efectos del diagnóstico, el suicidio no se considera necesariamente como el resultado de un trastorno psicopatológico.

Durkheim explica el suicidio como "todo caso de muerte que resulte, directa o indirectamente, de un acto, positivo o negativo, realizado por la víctima misma, sabiendo ella que debía producir ese resultado" (60). Usualmente se tiende a analizar cada caso de forma individual identificando causas muy puntuales para cada uno; sin embargo, para Durkheim, las verdaderas causas obedecen a fenómenos sociales. 
Por tanto, no se debe confundir el evento detonante del suicidio que puede dotarlo de ciertas particularidades con las verdaderas causas subyacentes. Como lo expresa el autor, "los más diversos acontecimientos de la vida hasta los más contradictorios pueden igualmente servir de pretexto al suicidio. Pero ninguno de ellos es su causa específica" (Durkheim 406).

El suicidio en una sociedad es interpretado, por Durkheim (341), como un hecho social que puede ser explicado a partir de las fuerzas sociales imperantes en esa sociedad, la cual ejerce cierto control sobre los individuos, pues "es el único poder moral superior al individuo, y cuya superioridad este acepta", de manera que esa determina en gran parte las acciones de sus integrantes, incluyendo el impulso de quitarse la vida.

En su análisis identifica tres tipos de suicidio de acuerdo con los diferentes controles morales de la sociedad, a saber: egoísta, altruista y anómico. El primero se caracteriza porque la persona no posee un vínculo con los grupos sociales que la rodean y, por tanto, carece de un sentido de vida al no poder cumplir con su fin social. Recibe el nombre de egoísta porque la individuación de la persona se vuelve excesiva. A partir de esto es importante señalar que, en el caso contrario, si la persona mantiene un vínculo con la sociedad se puede considerar un factor protector porque es capaz de encontrarle un sentido a la vida, al considerar que cumple con su papel social.

El suicidio altruista, por su parte, se da cuando la persona tiene una relación demasiado estrecha con sus grupos sociales, con lo cual pierde su individuación y se anula su personalidad. Este tipo de suicidio es común en sociedades pequeñas donde existe un fuerte control social y donde las personas viven las mismas circunstancias y condiciones, impidiendo así que el individuo se distancie del grupo.

Por último, el suicidio anómico es aquel que se da en sociedades desorganizadas, donde las reglas no logran regular las acciones de las personas y, por tanto, no existe una esfera social como en el suicidio egoísta; pero en este caso se vislumbra esa falta en la carencia de reglas para regular las pasiones individuales.

En momentos en que la sociedad es incapaz de mantener un equilibrio usualmente aumentan los suicidios, pues para el autor cada sociedad tiene una moral que combina el egoísmo, el altruismo y la anomia de forma equilibrada, pero si alguna de estas predomina las personas se hacen suicidógenas (Durkheim, 1974). Por tanto, se torna importante intentar comprender el contexto social y las fuerzas sociales en juego para explicar el suicidio, y a la vez trabajar con las instituciones y los grupos sociales para que, como fuerzas sociales, puedan contribuir a establecer el control necesario para evitarlo.

Un eje fundamental de la prevención del suicidio a nivel comunitario, desde un enfoque psicosocial, consiste en reconocer tanto los factores de riesgo, como los factores protectores presentes en una localidad en particular. Los primeros pueden elevar las probabilidades de que ocurran suicidios. Por su parte, los segundos son 
el conjunto de procesos sociales e intrasíquicos producto de la interacción entre la persona y su medio, los cuales pueden disminuir la probabilidad de ocurrencia de suicidios (Caycedo, Arenas, Benítez, Cavanzo, Leal y Guzmán, 2010; Navas, 2013; Andrade Salazar, Bonilla Gutiérrez y Valencia Isaza, 2010). Por tanto, en el marco del proyecto, es importante determinar ambos tipos de factores en las dinámicas comunitarias, con lo cual se pueden tomar acciones oportunas para minimizar ambos tipos de factores.

\section{Enfoque de salud comunitaria}

Según este enfoque, la salud está determinada por factores personales, familiares, socioeconómicos, culturales y físicos (Pasarín, Forcada, Montaner, De Peray y Gofin, 2010). El proceso salud-enfermedad desde:

La salud comunitaria incluye una concepción social de la salud, que tiene en cuenta el ámbito social en que se producen los fenómenos de salud-enfermedad, teniendo en cuenta los niveles macrosociales y microsociales, y la participación de las comunidades, instituciones y demás actores en la toma de decisiones (Pasarín et al. 10).

En otras palabras, en el abordaje de la salud no solo intervienen las instituciones tradicionales de la salud, sino también se toman en consideración otros actores de la comunidad, los cuales contribuyen a generar un estado de salud o enfermedad (Martín Zurro y Jodar, 2011). Por tanto, una de sus principales contribuciones consiste en redirigir el escenario donde se realiza la intervención al espacio comunitario y reconocer en las instituciones presentes un papel distinto asociado al tema de la salud.

Desde este enfoque, la salud no se limita a la ausencia de síntomas de enfermedad. Para mantenerla se requiere enfatizar en los determinantes sociales de la salud, los cuales corresponden a las condiciones de vida. Estas van desde su ambiente inmediato hasta políticas públicas (Pasarín et al., 2010) y pueden intervenir ya sea como factores de riesgo o como factores protectores (Martín Zurro y Jodar 2011). De esta forma, es fundamental conocer todos aquellos factores del medio que pueden incidir sobre el proceso salud-enfermedad, incluyendo la salud mental y, en este caso particular, el suicidio.

\section{Aspectos metodológicos}

El diagnóstico se realizó mediante una metodología cualitativa, pues esta es apropiada para profundizar en las nociones, percepciones y actitudes que mantienen las personas de la comunidad acerca de la problemática. Como lo explican Hernández, Fernández y Baptista (583) "esta clase de datos es muy útil para capturar de manera completa (lo más que sea posible) y sobre todo, entender los motivos subyacentes, significados y las razones internas del comportamiento humano". 
Por consiguiente, se utilizaron entrevistas, grupos de discusión y talleres participativos para recabar la información necesaria.

Al inicio, se realizaron 11 entrevistas individuales y 3 grupales no estructuradas. Debido a que el equipo de trabajo era ajeno a la comunidad y no se sabía si el tema del suicidio se podía hablar de forma abierta, se decidió iniciar con preguntas generales acerca de las problemáticas identificadas en la comunidad, especialmente aquellas relacionadas con la salud. El tema del suicidio salió a colación en prácticamente todas las entrevistas sin necesidad de preguntar de forma directa. Es importante aclarar que el objetivo de la investigación era identificar los factores con incidencia sobre la conducta suicida en Santa María de Dota. Por tanto, se consideró la perspectiva de las personas de la comunidad pero sin profundizar en aspectos personales o clínicos. Además, ni el riesgo de suicidio, ni el ser sobreviviente de suicidio fueron criterios considerados relevantes para la participación de las personas en el estudio.

En esta primera etapa no se grabaron las entrevistas, pero sí se tomaron apuntes. La sistematización de estas notas dio los insumos para elaborar las guías de los grupos de discusión y de las entrevistas realizadas en un segundo momento del trabajo de campo.

En este segundo momento se realizó un grupo focal con jóvenes de la comunidad. La intención era conformar dos grupos focales: uno de adolescentes y otro de adultos jóvenes. Sin embargo, el grupo de adultos jóvenes no se pudo constituir, de forma que se realizó una entrevista grupal semiestructurada y dos entrevistas individuales semiestructuradas para poder incluir su punto de vista. Por su parte, con el grupo focal de adolescentes se realizaron dos sesiones.

Tanto las conversaciones de los grupos focales como las entrevistas fueron grabadas, transcriptas y analizadas, lo cual dio como resultado distintos ejes temáticos relacionados con el suicidio en la comunidad, que serán expuestos en el próximo apartado.

Por último, se llevaron a cabo tres talleres participativos con diferentes poblaciones: mujeres, personas jóvenes e instituciones de la comunidad, con el objetivo de identificar propuestas de trabajo que puedan ser implantadas al siguiente año para prevenir el suicidio. Cada uno de los talleres inició con el análisis de la problemática a partir de la elaboración de un árbol de problemas, en el cual las personas identificaron las causas y consecuencias del suicidio. Luego, a partir de las causas se construyó otro árbol de soluciones que sirvió para hacer propuestas concretas a corto y mediano plazo para prevenir el suicidio.

Los resultados del diagnóstico surgen del análisis de las entrevistas no estructuradas y de las semiestructuradas, del grupo focal y de la construcción del árbol de problemas que se realizó en los talleres participativos. Por tanto, integra la opinión de quienes participaron en estos procesos de consulta, incluyendo adolescentes, adultos jóvenes, líderes comunitarios y representantes de instituciones. 


\section{Resultados del diagnóstico}

El análisis de los resultados se presentará en dos ejes. El primero contiene la caracterización del cantón de Dota, especialmente de su cabecera de cantón Santa María. Este primer eje expone las representaciones que tienen las personas acerca de las principales problemáticas y fortalezas de la comunidad, las cuales se interpretan como factores de riesgo o factores protectores en relación con el suicidio. En cuanto al segundo eje, este se enfoca en el análisis del suicidio propiamente, en especial sobre el conocimiento de las personas acerca del tema, los recursos de la comunidad para prevenir y abordar el suicidio, así como algunas estrategias generales mencionadas en los procesos de consulta para su prevención y abordaje.

\section{Caracterización del Cantón de Dota}

\section{Problemáticas de la comunidad entendidas como factores de riesgo}

En los diferentes procesos de consulta surgió una serie de problemáticas comunitarias que las personas vinculan con el suicidio, las cuales se pueden interpretar como factores de riesgo. Esas se agruparon en cinco temáticas generales, a saber: lo simbólico, la moral y los valores, el uso del tiempo, lo institucional y la vinculación, y la participación.

Figura 1

PROBLEMÁTICAS IDENTIFICADAS EN EL CANTÓN DE DOTA ASOCIADAS AL SUICIDIO

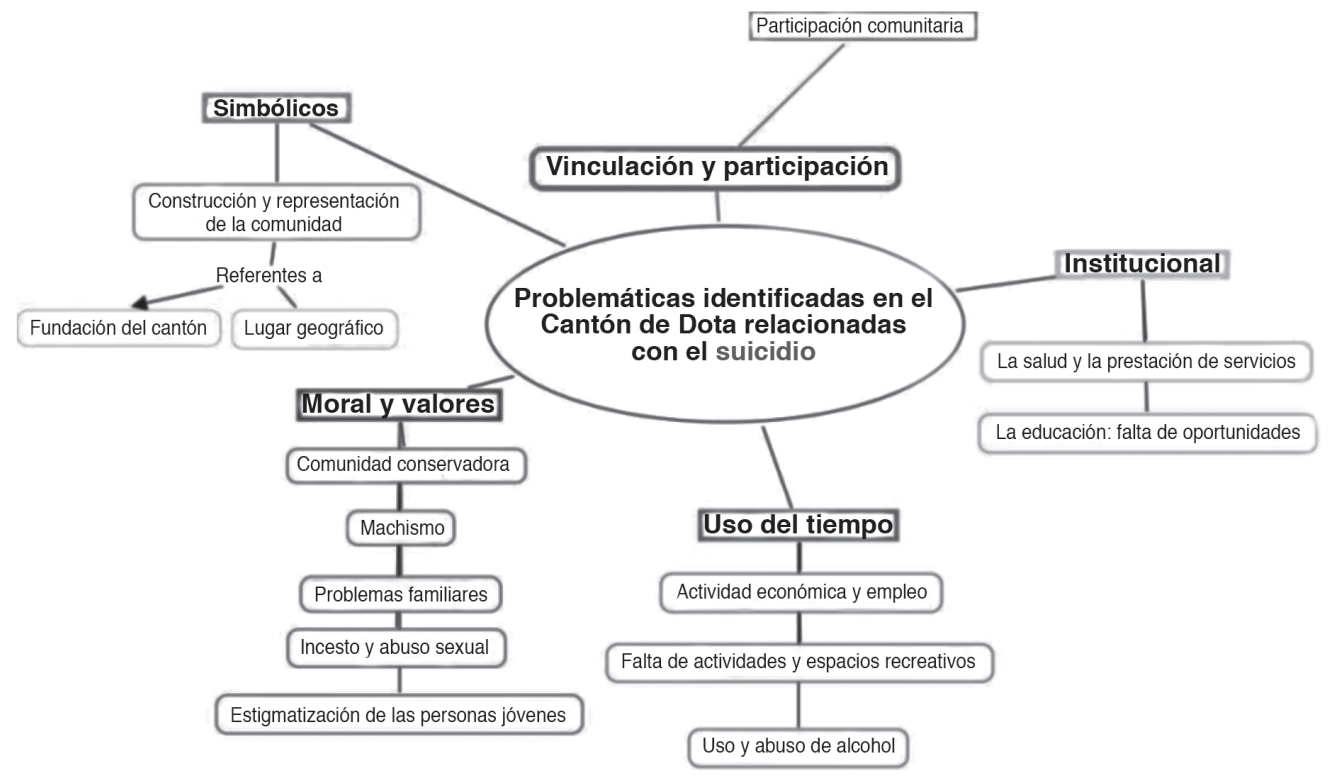

Fuente: Elaboración propia. 


\section{Lo simbólico}

Este aspecto se refiere a la representación simbólica que tiene la gente sobre el cantón, la cual gira entorno a dos aspectos: la fundación y la ubicación geográfica. Acerca de la primera es importante destacar que, según algunos líderes comunitarios, los actuales problemas de convivencia se remontan a las raíces históricas del cantón, pues entre las primeras familias asentadas en este territorio se produjeron conflictos y desavenencias, principalmente por la tenencia de la tierra; de manera que estas diferencias persisten hoy en día. Además, en la actualidad también se perciben discrepancias entre las familias originarias, cuyos apellidos son conocidos por todos, y las familias que se han asentado recientemente en el territorio. Como bien lo describe uno de los jóvenes entrevistados en el siguiente extracto:

También las divisiones que se dan en Santa María tal vez no es [sic] que usted las vea, pero sí la gente en el fondo sí sabe, di la gente sí clasifica definitivamente, yo sé por qué yo soy de ahí y todo el mundo lo sabe. La gente decide quien merece y quien no merece, iverdad? y son algunos los que dicen (Mauricio, 30 años).

Las diferencias también son explicadas en términos de una división de clases, pues se dice que las personas en la comunidad se preocupan mucho por mantener las apariencias aunque ello implique endeudarse. Al respecto, una joven adolescente comenta: "hay dos clases sociales: los que están endeudados y los que no lo estamos" (Cindy, 17 años).

Por su parte, en cuanto a la ubicación geográfica, las personas comentan que Santa María, al localizarse en un valle, genera la sensación de ser un lugar encerrado o encapsulado como se describe en el siguiente comentario: "Ustedes ven la forma es como sí cerrado, aquí hay muy poca actividad, aquí hay muy poco lugar para estudiar, la gente cuesta que se vaya para otro lugar, entonces como que la gente se encierra en el mismo pueblo" (Silvia, 27 años).

Estas características terrestres son interpretadas como barreras físicas que dificultan el poder visualizar un futuro o una realidad más allá de esos límites. Esto incide en la salud de la comunidad, pues impacta en su estado de ánimo. El siguiente extracto también hace alusión a esa sensación: "Es un valle, por lo cual las energías no fluyen y eso contamina la comunidad" (Isabel, mujer referente en la comunidad).

Además, Santa María de Dota es percibida como un ente con una personalidad propia distinta de quienes la habitan, ya que se habla como si fuera un elemento externo, el cual valoran en términos negativos. Esta situación la hacen explícita dos mujeres al afirmar que "Santa María está enferma".

Dicho aspecto es uno de los factores de riesgo más relevantes para el suicidio debido al impacto que tiene en los estados de ánimos, la sensación de encierro y la imposibilidad de ver un futuro más allá de los límites geográficos. 


\section{La moral y los valores}

Este segundo eje se compone de todo aquello relacionado con una moral conservadora y el mantenimiento de ciertos valores asociados al patriarcado, lo cual tiene un peso importante en las relaciones sociales, las prácticas económicas, las expresiones de violencia y el lugar que tiene la comunidad para los jóvenes, como parte de su percepción.

Dota se considera un lugar muy conservador regido por valores tradicionales; lo cual se observa principalmente en dos dimensiones: por un lado, se ubica lo relacionado con la moral, los valores y la religión; y, por el otro, el aspecto económico. En cuanto al primero, se tiene la idea de que es una comunidad donde existe una fuerte presión por cumplir con los principios morales predominantes. Se ejerce un fuerte control social especialmente a través del chisme, lo cual se identifica como un factor de riesgo, pues quien no se ajuste a los mandatos establecidos se puede convertir en tema de chisme, quedando expuesto a los juicios y críticas. Por tanto, se dificulta el establecimiento de redes de apoyo. Esto es expresado por gran parte de la población, pero especialmente son las mujeres y los jóvenes quienes lo vivencian, como lo demuestra el relato de esta joven:

Santa María de Dota es un pueblo para que me entienda, pueblo pequeño infierno grande, las personas, la mayoría de las personas aquí siempre señalan o nos señalan con cosas negativas, cuesta mucho que usted encuentra una persona que le dé a un apoyo moral por ejemplo o que lo haga a usted sentir bien, la mayoría y usted lo pueden preguntar aqui, lo que inventa son chismes y eso perjudica mucho y es en general (Pamela, 31 años).

Otra de las jóvenes expresó una percepción similar:

Es un pueblo demasiado, con una mente o una psicología muy, muy cerrada, muy machista, como que le gusta mucho señalar a la gente, juzgar, involucrarse en las vidas privadas, como que la misma gente se hace como mucho daño (Silvia, 27 años).

Un ejemplo concreto de expectativa moral es el cumplimiento de los papeles tradicionales de género, lo cual fue identificado como un problema por hombres y mujeres de distintas edades y ocupaciones. Algunas personas piensan que estos mandatos de género son reforzados por preceptos religiosos que se mantienen en las familias, pues la religión y los grupos religiosos aún tienen una fuerte presencia en la comunidad.

El machismo se refleja en las relaciones de poder y desigualdades de género que se dan tanto a nivel público como privado. Por lo tanto, se convierte en un factor de riesgo en tanto genera formas de opresión y violencia en contra de las mujeres; lo cual, aunado a las pocas posibilidades de denuncia y atención, se convierte en un factor de vulnerabilidad. En el caso de los hombres, se enfrentan a mandatos sociales 
establecidos, que si no pueden cumplir, retan su masculinidad hegemónica, a la vez que tienen posibilidades limitadas para expresar sus sentimientos de frustración y tristeza. Al respecto,

Ejemplo aquí si yo salgo mucho ya me ven como una mala mujer porque ando mucho en la calle. Ya la gente empieza hay vea es que esa, el cuchilleo verdad. En cambio, si es hombre no. Es un macho, anda en cantinas, anda con mujeres, como que al hombre le solapan más el hecho de que tenga una vida más liberal pero la mujer no (Silvia, 27 años).

El machismo también está asociado con situaciones de violencia intrafamiliar y abuso, las cuales se presentan al interior de las familias, y se relaciona, especialmente, con patrones de crianza. Si bien las personas reconocen que sucede con frecuencia y que desemboca en una desintegración familiar, no se habla públicamente sobre esto. Por ejemplo, según algunos líderes comunitarios, se han dado casos de incesto, pero se mantienen ocultos. Por su parte, este aspecto no es considerado como el factor de riesgo de mayor importancia, pero sí se le debe prestar atención debido a las secuelas, ya que puede desembocar en pensamientos suicidas en el agresor o la víctima, quien ante la falta de un apoyo oportuno puede desembocar en un suicidio consumado.

A partir de su estudio, Arias y Blanco (202) señalan que:

Relacionar cómo la recesión económica y el aumento del desempleo o la flexibilización del mercado laboral, así como las migraciones, la disminución del gasto público en salud y educación, y la mayor participación de la mujer en actividades remuneradas, pueden estar debilitando en algunas zonas rurales el protagonismo del hombre como sostén económico de la familia, erosionando a su vez su autoridad "moral" dentro de su grupo familiar.

Permiten explicar por qué se convierten en una población que tiene mayor vulnerabilidad frente al suicidio. Las personas de la comunidad también mencionaron que las familias tienen problemas de comunicación, sobre todo porque se les dificulta expresar sus sentimientos y emociones, lo cual se trata de solventar con "cosas materiales". Esto representa un factor de riesgo porque la familia en estos casos no es capaz de ejercer su papel de red de apoyo, ya que no puede ofrecer contención o seguimiento a sus integrantes. El siguiente extracto del grupo de discusión con adolescentes ejemplifica lo anterior:

-Si porque, digamos, yo he visto que también dicen, eh, yo le regalo un celular si usted se saca buenas notas (Catalina, 14 años). 
- Sí como que los compran, pero digamos eso no debería ser así, porque es el estudio y no debería de recibir nada (Daniela, 14 años).

-Digamos los compran entonces, ahi ya hacen lo que los papás quieren, y si logran conseguirlo empiezan a pedir más (Roberto, 16 años).

Al mismo tiempo, los jóvenes de la comunidad sienten un estigma hacia sus capacidades y actividades al ser tachados de "vagos", y sus actividades se ven señaladas al considerar que fomentan el uso y abuso de drogas y alcohol. Además, para ellos los grupos religiosos en conjunto con otras agrupaciones ejercen su influencia en las dinámicas y actividades comunitarias, al poner resistencia a la realización de ciertas actividades recreativas con el argumento de promover el consumo de alcohol. Esto incide como un factor de riesgo, pues limita las actividades recreativas realizadas en la comunidad, lo cual se convierte en un reclamo importante de los jóvenes. Ese estigma queda expuesto en el siguiente comentario: "Y la gente dice uy esos chiquitos que están ahí, que pereza. Que vagos, deberían de estar en la casa'” (Priscilla, 15 años). En este otro ejemplo:

Iban a hacer en la plaza, por donde uno espera el bus. El terreno lo arreglaron porque iban a hacer un skatepark para los muchachos de bicicletas y patinetas y cuando dijeron eso dijeron "ah no" ya los trataron de maleantes, de gente mala y de vagos y de todo, entonces no lo terminaron haciendo. Fueron a reclamar a la municipalidad y ya nunca lo hicieron (Aaron, 16 años).

En cuanto al aspecto económico, en la zona de los Santos se ha popularizado el siguiente dicho: "En Santa María la gente guarda, en San Marcos la gente invierte y en San Pablo la gente se divierte". En concordancia con este dicho, un común denominador entre las personas entrevistadas es que Santa María es descrita como la más conservadora y clasista de las tres cabeceras de cantón, lo cual ha obstaculizado, según se cree, el desarrollo del pueblo.

Tradicionalmente, Santa María se ha dedicado al cultivo del café como principal medio de subsistencia y de generación de empleo, ya que la tierra está bien repartida entre las familias residentes, permitiéndoles generar sus propios ingresos. Sin embargo, en la actualidad el negocio del café es más volátil que en años anteriores, lo cual ha llevado a los más jóvenes a buscar otras opciones de negocios o emprendimientos para subsistir e impulsar el desarrollo en la zona. No obstante, se han enfrentado a la negativa de "líderes viejos" que se resisten al cambio. Como consecuencia de esta cultura conservadora, se piensa que en Santa María cualquier idea de negocio o emprendimiento no tiene posibilidades de éxito por falta de apoyo y aceptación de la comunidad. 
En términos generales, la percepción de ese ambiente conservador y patriarcal es vivida por las personas desde un fuerte control social. Cada grupo lo experimenta de diferentes maneras; por ejemplo, las personas jóvenes se sienten excluidas; las mujeres lo vivencian a través de diferentes formas de violencia y censura, y los hombres sienten la presión de cumplir con la expectativa del mandato de papel hegemónico.

Según Durkheim, la sociedad tiene el papel de regular la moral como un mandato con un poder superior al del individuo. Además, para él, cuando estos mandatos buscan una fuerte integración de las personas, dejando poco lugar a la disidencia en el pensamiento, las ideas, las creencias y las conductas, los suicidios se pueden explicar desde lo que él denominó suicidio altruista, pues en este caso la individualidad pierde sentido y valor frente a lo colectivo. Sin embargo, el análisis no estaría completo sin la referencia al suicidio anómico, el cual se caracteriza por la falta de soporte; aunque en este caso no se trata de una ausencia de normas, sino de una falta de soporte en las familias debido a los problemas de desintegración y a las limitaciones para la comunicación y el acompañamiento emocional que las personas consultadas identifican.

\section{Uso del tiempo}

El siguiente factor de riesgo es el uso del tiempo, pues las personas de la comunidad reconocen que se tiene mucho tiempo libre y pocas actividades en las cuales aprovecharlo, lo cual tiene una incidencia en ciertas problemáticas de la comunidad incluyendo el suicidio.

Una de las principales problemáticas identificadas por las personas en Dota es el desempleo; no obstante, este no se reconoce como un factor de riesgo para el suicidio por la dimensión económica, sino por la cantidad de tiempo libre que tienen las personas. Pérez (95) afirma que "los cambios económicos no solo influyen en el orden de los costos y ganancias, también modifican los ritmos de vida, las costumbres y la incorporación de nuevos elementos en la vida cotidiana".

Al ser la producción de café la principal actividad económica del cantón, durante los meses de diciembre, enero y febrero las personas se encuentran ocupadas y cuentan con un sueldo. Por tanto, es la época más dinámica en términos económicos, mientras que el resto del año la situación se pone más "dura"; pues en el invierno, con las lluvias, la jornada de trabajo se acorta y las personas tienen más tiempo libre. Por esta razón, uno de los líderes comunitarios opina que "la cultura del suicidio en Dota está muy relacionada con la cultura del café" (Miguel, 55 años). Esto, junto con la falta de actividades y espacios recreativos, favorece que las personas se encierren en sus problemas, dando cabida a la depresión y a la ideación suicida. Al mismo tiempo, otras posibilidades de empleo o de desarrollo profesional son muy limitadas, especialmente para personas jóvenes o mujeres amas de casa. Por esta razón, muchas personas jóvenes buscan migrar a San José en busca de otras oportunidades laborales. 
Para las personas jóvenes, el tema de las actividades y los espacios recreativos es fundamental, pues la falta de estos afecta a toda la comunidad, pero principalmente a los niños, niñas y adolescentes por lo que se convierte en un tema prioritario para las poblaciones. Si bien en la comunidad se identifica la existencia de espacios para la recreación como la plaza y el gimnasio, estos no pueden ser utilizados libremente por los jóvenes, en especial porque los grupos que los administran limitan el acceso:

Imaginese que a las 5:30 uno llega a la plaza y más bien empieza a sacar a todo mundo, no tiene los marcos puestos, y en esas reglas está de que si llovió no pueden entrar, y no se puede entrar con mascotas, o sea, no se puede nada (Roberto, 16 años).

Por su parte, hay una oferta pequeña de grupos culturales como el coro, la sinfónica o los bailes típicos, pero estos no tienen un alcance tan amplio y en ocasiones requieren de una inversión económica que limita la posibilidad de participar a muchas personas.

Como consecuencia, los pobladores se encierran en sus casas frente al televisor o los bares se convierten en su principal actividad recreativa, lo cual estimula el consumo de alcohol y de drogas. Esto se da de forma regular en todas las edades, convirtiéndolo en un factor de riesgo importante:

En realidad aqui en Santa María usted le puede preguntar a los jóvenes y ellos le van a contestar que aqui no hay nada que hacer y yo les comenté a ustedes que lo que aqui había siempre, eran bares, eso es lo que hacen. Los fines de semana lo que hacen aquí los jóvenes es ir a los bares, porque digamos entonces yo pienso que hace falta como abrir esos espacios donde usted participe, donde usted deje la vergüenza porque hay muchos jóvenes que no lo hacen por vergüenza talvez, que brincar mecate, jay no, qué vergüenza!, o así digamos, tener participación, aprender a que esos, a crear una comunidad con esa mentalidad, digamos que no solo para los bares, eso no es solo la diversión (Pamela, 31 años).

Otros estudios sobre el tema del suicidio en comunidades rurales e indígenas en América Latina, más allá de las explicaciones particulares para este tipo de casos, coinciden en que existe una tendencia al aumento del número de suicidios cuando las personas enfrentan cambios importantes, sea debido a situaciones de desplazamiento y violencia (por ejemplo en Colombia), por modificaciones estructurales producidas en las actividades productivas o por el encuentro abrupto con la sociedad occidental, en el caso de las comunidades indígenas (Arias y Blanco 188; Pérez 98; Sepúlveda López de Meza 259; UNICEF 198). 


\section{Institucional}

El cuarto tema por analizar es el relacionado con las carencias institucionales, especialmente aquellas referidas al sector salud y educación. Las familias valoran el estudio y procuran ofrecer esta oportunidad a sus hijos (incluyendo el nivel universitario), quienes lo consideran una herramienta útil para sus vidas y lo visualizan como un espacio para relacionarse con sus pares, pero también destacan que en los colegios se da mucha violencia o acoso escolar, lo cual se convierte en un factor de riesgo para adolescentes que enfrentan situaciones de este tipo y, a la vez, reciben poco apoyo.

En cuanto a la oferta de estudio, los jóvenes valoran positivamente el contar con un colegio técnico en su comunidad, pero consideran que la calidad de la educación ha disminuido. Además, para ellos la oferta de la educación universitaria es reducida, lo cual provoca que muchos de ellos se trasladen a otros lugares del país para recibir esta instrucción. Esto lo perciben como un inconveniente, pero no representa un factor de riesgo prioritario para el suicidio.

En relación con el sector salud, las personas consideran que no existe una adecuada labor preventiva y de promoción de la salud, especialmente mental, pues se entiende que no se limita a la condición física, sino también incluye mental, esta última vinculada con el suicidio.

En el caso de Santa María, para las personas del lugar uno de los problemas principales es que el EBAIS tiene una constante rotación de personal, lo cual provoca la ausencia de un compromiso real con la comunidad y la falta de una adecuada atención a los temas prioritarios, entre ellos el suicidio y la depresión. Además, en el Área de Salud Los Santos solo hay dos profesionales en psicología para atender a toda la zona; de esta manera, la atención en salud mental es limitada, pues es percibida de forma negativa por el estigma que persiste sobre la atención psicológica.

De acuerdo con la información recopilada en los talleres, la población consume altas cantidades de fármacos, lo cual se debe a la necesidad de recetar medicamentos ante la limitada atención en salud mental, especialmente para padecimientos como la depresión. A esto se le debe aunar el hecho de que durante las visitas a los hogares, el personal de salud ha encontrado muchos casos de aislamiento y soledad, especialmente en adultos mayores.

Estas deficiencias en el sector salud son un factor de riesgo de mucho peso para el suicidio, principalmente por la falta de posibilidades en la comunidad de dar atención y seguimiento a personas en riesgo suicida y porque la respuesta, en la mayoría de los casos, consiste en "medicalizar" sin promover una resolución adecuada de los problemas. Al respecto, en los países desarrollados el aumento en el consumo de antidepresivos coincide con el agotamiento de las políticas de atención en salud mental (Martínez 45). Al considerar esta premisa como referencia, se puede afirmar que en un 
sistema de salud pública como el costarricense, en donde la salud mental no es prioritaria en el abordaje (lo cual se agrava en zonas rurales donde el acceso a estos servicios es más limitado), el riesgo suicida no logra un acompañamiento integral, sino que su abordaje se centra en la atención de la emergencia. Martínez (49) denomina esto como "fetichismo de la enfermedad", pues la aflicción se reduce a su dimensión biológica-fisiológica como si no tuviera ningún vínculo social, y por lo tanto es atendida como tal.

\section{Vinculación y participación}

Este último eje se refiere a cómo se percibe la participación comunitaria. Se piensa que es una comunidad muy solidaria en eventos particulares como apoyo económico a la iglesia o durante procesos de duelo, pero en otros momentos constituye una comunidad resistente a participar en otros procesos. Por esta razón, se hizo referencia a algunas iniciativas que no se han podido llevar a cabo debido a la falta de apoyo o colaboración.

Si bien este no es un factor de riesgo relacionado con el suicidio, sí es uno importante para el proyecto debido al enfoque participativo y de gestión comunitaria que lo caracteriza; de esta manera, se debía considerar para la propuesta e implementación de estrategias preventivas del suicidio.

\section{Fortalezas de la comunidad entendidas como factores protectores}

Es importante mencionar que en un primer momento los aspectos indicados en los procesos de consulta fueron las problemáticas; sin embargo, las personas también manifestaron sentir mucho apego e identificación con su comunidad cuando se les preguntó por los aspectos positivos o fortalezas.

En primer lugar, lo caracterizan como un lugar muy tranquilo y seguro, razón por la cual les gusta vivir ahí. Segundo, cuentan con redes de apoyo al tener a la mayoría de los familiares viviendo en el cantón; así a pesar del problema de los chismes, las personas consideran como un apoyo tener a la familia cerca. Por último, se menciona el aspecto natural destacando los espacios verdes donde se pueden realizar actividades, respirar aire puro y convivir con un entorno "muy verde".

Estos dos últimos aspectos positivos en contraste con los aspectos negativos o factores de riesgo podrían parecer contradictorios; sin embargo, demuestran una gran ambivalencia, pues pueden ser vistos como factores de riesgo o factores protectores. Por ejemplo, en algunos casos, el vivir en un pueblo pequeño donde existe una gran cercanía entre sus habitantes se percibe, por un lado, como un fuerte control social; mientras que, por el otro, se considera como una red de apoyo. Asimismo, el entorno natural puede dar la sensación de ahogo o encierro, o bien ser el escape frente al estrés o el aburrimiento. De cualquier manera, en términos del diagnóstico, lo importante fue evidenciar que las problemáticas o factores de riesgo exceden en 
cantidad y en orden de importancia a los aspectos positivos o fortalezas. Por lo tanto, el trabajo preventivo debe ir encaminado a disminuir los factores de riesgo y potenciar los factores protectores.

\section{El suicidio en Dota}

En este segundo eje se expone la percepción y el conocimiento que tienen las personas consultadas acerca del suicidio, así como las herramientas o las estrategias presentes en la comunidad para hacerle frente en cada una de las etapas del suicidio.

El suicidio es un tema de importancia para los diferentes sectores de la comunidad participantes en el estudio. No obstante, la mayoría reconoce no hablar de este tema abiertamente, excepto cuando ocurre un suicidio, en cuyo caso se trata, principalmente, como un chisme. Esto incide en la percepción de que este no es prioritario en la comunidad al no generarse una discusión sobre cómo prevenir nuevos casos o abordar los existentes:

Como lo expresa este joven: Porque a la gente no le gusta tocar eso, prefiere hablar de otras cosas. Prefiere hablar de religión, fútbol, eso es lo que, si o ir a tomarse las birras. Pero síno son temas que por lo general se toquen (Mauricio, 30 años).

Una opinión similar es mencionada por este participante: "Yo pienso que es un tema muy cerrado. No se habla para nada la verdad" (Rolando, 21 años).

Aparte de los factores de riesgo comunitarios señalados en el apartado anterior, también se reconocieron otros factores sociodemográficos. La literatura evidencia diferencias en cuanto al suicidio dependiendo del género y la edad (Organización Mundial de la Salud, 2000), las cuales son observadas en Santa María de Dota. En esta comunidad los hombres se suicidan más que las mujeres, lo cual es explicado por las personas a partir de la construcción de género, pues los hombres no sienten la misma libertad para expresar sus emociones tan abiertamente como las mujeres.

Porque el hombre y más en un, es un lugar bastante machista, ¿verdad? Donde el hombre no tiene derecho a expresarse porque si no sería un afeminado o no sé, no se puede expresar tan libremente, ¿verdad? Llega a ser un amargado si llega o no sé, igual yo pienso que si quiere ser un amargado que lo sea, no me importa ¿entiende? Tiene libertad de serlo, ahí no está esa libertad. Es que la gente, solo el hecho de eso ya es una represión, es algo que (Rolando, 21 años).

En cuanto a la edad, para los adolescentes y los adultos jóvenes la etapa de la adolescencia es un periodo de riesgo porque se comienza a tomar conciencia de las presiones sociales y por tanto las personas se vuelven más susceptibles a los juicios y las críticas. No obstante, el suicidio consumado ocurre más en personas jóvenes adultas. 
Estos factores de riesgo aunados a otros factores personales o psicológicos pueden profundizar los sentimientos de soledad o de aislamiento poniendo a la persona en mayor riesgo (Caycedo et al. 7). Como consecuencia, la desesperanza se considera como un aspecto de gran trascendencia, pues si la persona tiene posibilidades de salir adelante y cuenta con un proyecto de vida hay una mayor posibilidad de que le haga frente a sus problemas, pero en caso contrario existe un gran riesgo de suicidio.

Las personas también identificaron factores protectores frente al suicidio (Navas 22); por ejemplo, la familia, pues puede brindar apoyo y espacio para comunicarse. No obstante, la red de apoyo puede estar integrada por otras personas externas a ese círculo. Lo importante en este caso es reconocer el valor de una red de apoyo al considerársele como un factor protector, ya que para el proyecto esto se convierte en uno de los elementos claves para trabajar el suicidio desde el enfoque psicosocial: "la familia es fundamental, porque el apoyo familiar es algo que nos alimenta a hacer mejor las tareas" (Pamela, 31 años).

Otro factor protector es la dimensión espiritual o religiosa, pues se piensa que creer en un ser superior puede dar sentido a la vida, así como adquirir herramientas para enfrentar los problemas. Sin embargo, se considera un factor de riesgo cuando se dice que "la falta de Dios" lleva a una persona a un estado de mayor vulnerabilidad. Por último, se hizo referencia a la importancia de contar con factores protectores personales, es decir, aquellas cualidades individuales que les facilitan a las personas enfrentar situaciones problemáticas de la forma más adecuada posible.

\section{Etapas del suicidio}

Al hablar de prevención del suicidio es importante conceptualizar la conducta suicida como un proceso que consta de diferentes momentos por los cuales puede pasar una persona, ya que estos pueden tomarse como una ventana de tiempo en la cual si se actúa de forma oportuna se puede prevenir. Este no necesariamente sigue un curso lineal hasta desembocar en el suicidio consumado, sino que puede haber alternancia entre los momentos anteriores.

El suicidio en la mayoría de los casos no constituye un acto espontáneo, de tal manera, identificar el momento en el que se encuentra la persona permite valorar el nivel de riesgo y con base en eso definir el curso de acción adecuado para prevenirlo.

A continuación se expone la percepción de las personas con respecto a cada uno de los momentos, incluyendo las herramientas que existen en la comunidad para hacerle frente a cada uno.

\section{Ideación suicida}

El primer momento corresponde al pensamiento suicida, el cual se caracteriza por tener pensamientos acerca del suicidio. Este puede variar en intensidad y elaboración, 
ya que se puede pensar acerca del suicidio de forma ocasional o permanente, así como puede pensarse de forma general o con cierto nivel de detalle. De cualquier forma, estos pensamientos deben tomarse como predictores del suicidio (Baltodano Chacón y Márquez Cueva 2).

De acuerdo con las consultas realizadas, la ideación suicida es común en la comunidad y ha tendido a incrementarse entre las personas jóvenes. Por su parte, para el grupo de adolescentes no existen diferencias en cuanto al género; sin embargo, las personas jóvenes adultas piensan que es más común en hombres, debido a la falta de libertad para expresar sus emociones.

Si bien la literatura menciona que las personas en riesgo de suicidio usualmente transmiten advertencias verbales o de comportamiento acerca de sus pensamientos o intenciones (Organización Mundial de la Salud 15), para las personas consultadas no todas dan este tipo de advertencias, lo cual no hace posible percatarse en todos los casos si alguien está teniendo pensamientos suicidas. En su experiencia, las personas cercanas a individuos que se han suicidado no captaron ninguna señal o advertencia, por tanto, no tenían seguridad de identificar una persona en riesgo si esta no lo dice directamente.

Con respecto al abordaje de casos con pensamiento suicida, las personas afirmaron no saber cómo hacerle frente a un caso de este tipo, pues existe una gran incertidumbre sobre la forma adecuada de proceder o de brindar apoyo a la persona; incluso, algunos manifestaron que sentirían temor. Además, a nivel comunitario no existen mecanismos para abordar estos casos, lo cual provoca que la responsabilidad recaiga sobre ellos.

A pesar de su incertidumbre, se indagó cuáles serían sus respuestas ante un caso de ideación suicida; estas fueron de dos tipos. La primera consistía en buscar a alguien de confianza con una mayor capacidad para atender la situación, y la segunda proponía brindar acompañamiento a esa persona, al darle la oportunidad de hablar sobre su situación y buscarle actividades para que encuentre nuevamente un sentido a su vida.

Aparte de estas acciones, se considera que la persona en riesgo debería buscar la ayuda de un profesional de la salud, preferiblemente en psicología. Sin embargo, se reiteró que en la comunidad el acceso a este recurso, ya sea público o privado, es limitado, lo cual no es una alternativa para algunas personas.

\section{Gesto o intento suicida}

El segundo momento corresponde al gesto o intento suicida, lo cual implica que la persona ya realizó alguna acción en contra de su vida. Entre ambos conceptos hay diferencia, pues el gesto se entiende como aquella acción contra la propia vida 
mediante métodos no letales, y cuya posibilidad de acabar en la muerte es reducida; por su parte, el intento se comprende como el uso de métodos letales con intenciones y alta probabilidad de acabar con la vida, pero que por otras razones no sucede (Tapia, Vohringer, Ornstein 188).

De acuerdo con el Ministerio de Salud (183), Dota se encuentra entre los primeros cantones a nivel nacional con una de las tasas más altas por intento de suicidio. Por tanto, aunque ocurre constantemente, el gesto o intento de suicidio se silencia en la comunidad, lo cual dificulta estimar su frecuencia. Sin embargo, se percibe que son los adultos jóvenes quienes lo intentan más, mientras que los adolescentes solo lo piensen.

Un aspecto a resaltar en los resultados del diagnóstico fue la frecuencia con la cual las personas adolescentes incurren en conductas de riesgo, por ejemplo cortarse o golpearse contra la pared. En el grupo focal con adolescentes se discutió este tipo de conductas autolesivas. Comparativamente resulta más común en hombres que en mujeres. Para algunos participantes, estas conductas tienen el propósito de llamar la atención, pero, al mismo tiempo, no descartan que algunos lo hacen para manifestar sus sentimientos como el enojo o la frustración, lo cual puede convertirse en una conducta de riesgo.

Por la falta de conocimiento acerca de los gestos o intentos de suicidio en la comunidad, las personas consultadas no pudieron indicar si es más frecuente en hombres o en mujeres. No obstante, los hombres utilizan métodos más letales, como el consumo de agroquímicos o la asfixia; mientras que las mujeres emplean métodos menos efectivos, como las pastillas; esto lleva a pensar que son ellas quienes lo intentan con mayor frecuencia.

A su vez, una persona puede pasar del pensamiento suicida al gesto o intento de suicidio por la combinación de ciertos factores de riesgo personales y la falta de factores protectores, de tal manera se pueden desencadenar o profundizar sentimientos de desesperanza. Por tanto, se recalcó la importancia de contar con factores protectores como las redes de apoyo y el fortalecer los factores protectores personales para prevenirlo.

Por último, frente a un caso de gesto o intento de suicidio, los participantes manifestaron sentir la misma incertidumbre porque no sabrían cómo proceder, aunque su primera opción sería conversar con la persona implicada, seguido de buscar ayuda espiritual, moral o psicológica. Sin embargo, mencionaron nuevamente las limitaciones de la comunidad como la falta de acceso a estos recursos y la imposibilidad de encontrar el apoyo de la comunidad debido a las dinámicas sociales negativas que la caracterizan, como el juicio, los chismes, la división social, entre otros.

A pesar de que existe consenso de que casos requieren de un apoyo y seguimiento más cercano, en Dota las personas no están capacitadas para dar ese acompañamiento y las instituciones tampoco ofrecen alternativas de referencia o atención. 


\section{Suicidio consumado}

Por último, se puede hablar del suicidio consumado como el momento en el cual la persona logró quitarse la vida. En Dota, al igual que sucede nacional e internacionalmente, el suicidio consumado se da con más frecuencia en hombres que en mujeres, lo cual se puede explicar a partir de los mandatos de género presentes la comunidad. Además, según los participantes, el suicidio es menos frecuente en las mujeres por el tipo de métodos que utilizan, los cuales permiten intervenir de manera oportuna a otras personas con el fin de evitar que la situación desemboque en un suicidio consumado.

Aunque a nivel comunitario los suicidios se hablan en tono de chisme y esto contribuye a la percepción de que la comunidad no se ve afectada, los participantes expresaron sentirse afectados emocionalmente, en especial si el caso es cercano. Los sentimientos identificados son diversos, a saber: tristeza, arrepentimiento, impotencia, culpa y preocupación por las personas allegadas al difunto.

En cuanto a las consecuencias del suicidio, para las personas consultadas los mayoritariamente afectados son la familia y las personas más cercanas. Piensan que las afectaciones pueden ser sobre todo emocionales o psicológicas, pero también se ven afectadas cuando deben asumir las responsabilidades de la persona fallecida en casos donde, por ejemplo, tenían deudas u otros dependían económicamente de ella. Por tanto, los participantes coinciden en que no es una estrategia adecuada para solucionar los problemas.

Además, otra consecuencia puede deberse a que algunas personas tomen el ejemplo del suicidio como una alternativa para salir de los problemas, lo cual se convierte en una conducta aprendida; como mencionan Carmona Parra et al. (2010). En referencia a esta idea, uno de los entrevistados utilizó el término "cultura del suicidio" para explicar la situación presente en la comunidad. Asimismo, otras personas advierten la recurrencia de suicidios en ciertas familias a partir de este "aprendizaje" de la conducta o porque se presentan ciertos patrones de crianza negativos o violentos, los cuales posteriormente desencadenan otros problemas y aumentan la probabilidad de suicidios. En estos casos, la familia contraria a jugar su papel como factor protector se convierte en un factor de riesgo.

\section{Estrategias propuestas para la prevención}

Si bien en la comunidad actualmente no existen estrategias para prevenir el suicidio, las personas destacan la importancia de trabajar el tema y establecer medidas para evitarlo. En el caso de los jóvenes, sus propuestas de prevención van dirigidas a enfrentar las problemáticas que más los aquejan, por ejemplo la falta de espacios y actividades recreativas. De esta forma, sus propuestas van en la línea de la ampliación de estas, así como promover la participación de los jóvenes en grupos de toma de decisiones. 
A la vez, se mencionó la importancia de abrir espacios para hablar sobre el tema. Por tanto, una propuesta es la creación de un grupo de apoyo para sobrevivientes y otro para personas que se encuentran en riesgo, de manera que cuenten con espacios seguros donde puedan hablar abiertamente sobre su situación.

En los procesos de consulta con instituciones y con el grupo de mujeres surgió la necesidad de conocer más acerca del suicidio y de otros temas relacionados. Por tanto, las instituciones solicitaron capacitar a su personal, mientras que el grupo de mujeres pidió recibir charlas o talleres al respecto.

Además, ante la falta de estrategias para dar acompañamiento a las personas en riesgo y a sus familias se propuso crear una red compuesta por las instituciones y personas de la comunidad que se guíe por medio de un protocolo con el fin de dar apoyo oportuno a quienes lo requieran.

\section{Conclusiones}

Los enfoques utilizados para estudiar el suicidio tradicionalmente, los cuales se centran en factores individuales y clínicos, se tornaron insuficientes para comprender la alta tasa de suicidios de una localidad en particular, como es el caso de Dota. Por tanto, para considerar aquellos factores sociales y comunitarios incidentes en este conjunto de problemas, fue necesario partir de otros enfoques más integrales como el psicosocial y el de salud comunitaria, ya que permiten entender el suicidio como un fenómeno social producto de una conjunción de factores diversos, los cuales varían de acuerdo con la localidad en estudio. Asimismo, para elaborar el diagnóstico se utilizó una metodología cualitativa y participativa que incluyó entrevistas, grupos de discusión y talleres, partiendo de que quienes mejor conocen a su comunidad y sus problemáticas son sus pobladores.

A partir de estos procesos de consulta con líderes de la comunidad, mujeres, adolescentes, jóvenes adultos y personal de instituciones, fue posible elaborar un diagnóstico comunitario que integró la percepción de todos ellos acerca de los principales problemas de la comunidad y sus fortalezas o aspectos positivos, así como su percepción del suicidio y las herramientas con que cuenta la comunidad para hacerle frente.

Una de las principales conclusiones a las que se pudo llegar con este diagnóstico es que las personas de la comunidad no relacionan la patología mental o factores individuales con el suicidio; como lo sugieren los enfoques tradicionales, sino que existe una relación más directa con otros factores o problemas comunitarios, los cuales, desde su punto de vista, pueden incidir en la tasa de suicidio.

Por tanto, problemáticas como las críticas y juicios de la comunidad, los problemas económicos, la falta de empleo, la construcción simbólica de la comunidad como un lugar cerrado o encapsulado, los problemas familiares, el machismo, la falta 
de espacios y actividades recreativas y el pensamiento tradicionalista que se resiste al cambio constituyen particularidades de Santa María que, quizás, pueden incidir en la ocurrencia de casos de suicidio en la localidad. No obstante, es importante mencionar que no a todos se les otorga el mismo peso como factores de riesgo.

Esta clasificación de los factores (Tabla 1) que tienen más o menos peso como factores de riesgo para el suicidio no se basa en una escala de medición, sino en la valoración cualitativa hecha a partir de la información obtenida. Por tanto, no implica que necesariamente haya consenso total sobre estas apreciaciones, pero sí son aspectos que, de acuerdo con la mayoría de las personas consultadas, podrían tener una mayor o menor incidencia en la prevención del suicidio.

Tabla 1

FACTORES DE RIESGO RELACIONADOS AL SIUCIDIO Y SU CLASIFICACIÓN

Factores más importantes relacionados c on el suicidio
Factores menos importantes relacionados c on el suicidio
1. Construcción y representación simbólica de la comunidad.
2. Comunidad conservadora.
3. Machismo.
4. Estigmatización de las personas jóvenes.
5. Problemas familiares.
6. Falta de actividades y espacios recreativos.
7. Uso y abuso de alcohol.
8. La salud y la prestación de servicios.

Fuente: Elaboración propia.

También, se identificaron aspectos positivos en la comunidad, los cuales se consideran factores protectores frente al suicidio, entre estos: la seguridad y tranquilidad, el entorno natural y las redes de apoyo. No obstante, lo que resulta de importancia para este análisis es que para las personas, las problemáticas de la comunidad (factores de riesgo) exceden en cantidad y en relevancia los aspectos positivos (factores protectores). Por tanto, este mapeo no solo permite comprender la problemática del suicidio, sino también establecer y delinear estrategias de trabajo para fortalecer los factores protectores y disminuir los de riesgo.

Por último, a pesar de que el suicidio es una problemática presente en la cotidianidad del cantón, este no se ha considerado prioritario puesto que no existen 
los mecanismos adecuados para su referencia, atención y seguimiento, mucho menos estrategias para su prevención. No obstante, los participantes consideran que es importante establecer medidas preventivas, pues existe gran incertidumbre acerca de cómo proceder cuando se presentan casos de personas en riesgo en cualquiera de los momentos previos al acto de suicidio.

\section{Bibliografía}

Andrade Salazar, José A.; Leidy L. Bonilla Gutiérrez y Valencia Isaza Zully M. "Factores protectores de la ideación suicida en 50 mujeres del Centro Penitenciario:" Villa Cristina" Armenia-Quindío (Colombia)". Orbis. Revista Científica Ciencias Humanas, 6 (17) (2010), pp. 6-32.

Arias, Eliecer e Iraida Blanco. “Una aproximación al entendimiento del suicidio en comunidades rurales y remotas de América Latina". Estudios Sociológicos, XXVIII, (82) (2010), pp. 185-210.

Baltodano-Chacón, Maureen y Miguel Márquez-Cueva. "Ideación suicida en privados de libertad: Una propuesta para su atención". InterSedes, 15 (32) (2014), pp. 223-248.

Carmona Parra, Jaime A.; Felipe Torbón Hoyos, Juan C. Jaramillo Estrada y Yuliana A. Areiza Sánchez. El suicidio en la adolescencia en la pubertad y la adolescencia. Un abordaje desde la Psicología Social. Medellín: Fondo Editorial Funlam, 2010.

Caycedo, Alejandra; María L. Arenas, Michelle Benítez, Paula Cavanzo, Gretel Leal y Yahira Guzmán. “Características psicosociales y familiares relacionadas con intento de suicidio en una población adolescente en Bogotá-2009". Persona y BioÉtiCa, 14 (2) (2010), pp. 205-213.

Chaves Moreno, Allan; Edgar Madrigal Ramírez, Maikel Vargas Sanabria y Zarela Abarca Villalobos. El suicidio en Costa Rica en el año 2005. Medicina Legal de Costa Rica, 25(1) (2008), 17-35.

Durkheim, Emile. El suicidio. Ciudad de México: Universidad Nacional Autónoma de México, 1974.

García Alba, Javier E.; Roque Quintanilla Montoya, Luis M. Sánchez Loyo, Teresita Morfín López y José I. Cruz Gaitán. “Consenso Cultural sobre el Intento de Suicidio en Adolescentes". Revista Colombiana de Psicología, 20(2) (2011), pp. 167-179.

Hernández, Roberto; Carlos Fernández y Pilar Baptista. Metodología de la Investigación. México: Editorial McGraw Hill Interamericana, 2006.

Martín Zurro, Armando y Gloria Jodar. Atención familiar y salud comunitaria. Barcelona: ELSEIVER, 2011.

Martínez, Ángel. “La mercantilización de los estados de ánimo. El consumo de antidepresivos y las nuevas biopolíticas de las aflicciones". Política y Sociedad, 43 (3) (2006), pp. 43-56.

Ministerio de Salud. Indicadores Básicos 2013: Situación de la salud en Costa Rica. Dirección de Vigilancia de la Salud. San José: Ministerio de Salud, 2013.

Ministerio de Salud. Análisis de la Situación de Salud de Costa Rica. Dirección de Vigilancia de la Salud. San José: Ministerio de Salud, 2014.

Moscovici, Serge. La representación social: un concepto perdido. El Psicoanálisis, su imagen y su público. Buenos Aires: Editorial Huemul, 1979.

Navas Orozco, Wendy. "Abordaje del suicidio en atención primaria". Revista Médica de Costa Rica y Centroamérica, 70(606) (2013), pp. 219-225. 
Organismo de Investigación Judicial. Anuario Policial 2013. Departamento de Planificación, Sección de Estadística. Web. 16 de junio del 2016. http://www.poderjudicial.go.cr/ planificacion/images/documentos/estadisticas/policiales/Anuario_Policial_2013/ Elementos/PDFs/09_sumario_suicidios.pdf

Organización Mundial de la Salud. Prevención del Suicidio: Un instrumento para trabajadores de atención primaria en salud. Ginebra, 2000. http://www.who.int/mental_health/ media/primaryhealthcare_workers_spanish.pdf

Organización Panamericana de la Salud. Mortalidad por suicidio en las Américas. Informe regional. Washington, DC.: Organización Panamericana de la Salud, 2014.

Organización Panamericana de la Salud. Prevención del suicidio. http://www.who.int/mental_health/prevention/es/

Organización Panamericana de la Salud. Suicidio en Centroamérica y República Dominicana. Informe Subregional del Suicidio. Panamá: Organización Panamericana de la Salud, 2011.

Pasarín, María I.; Carmen Forcada, Isabel Montaner, Josep L. De Peray y Jaime Gofin. “Salud comunitaria: una integración de las competencias de atención primaria y de salud pública. Informe SESPAS 2010". Gaceta Sanitaria, (dic 2010), pp. 23-27.

Pérez, Andrea. “Muertes silenciadas: Problemática del suicidio en los campesinos de La Unión (Antioquia)". Revista Nacional Facultad de Salud Pública, 32.2 (2014), pp. 92-102.

Sáenz Rojas, Mario. “Aportes para una epidemiología del suicidio en Costa Rica: un análisis de la primera década del siglo XXI". Revista Ciencias Sociales, 131-132 (I-II) (2011), pp. 37-55.

Sánchez Martínez, Fernando y Guillermo Barrientos. Psiquiatría Social y Comunitaria. Santo Domingo: Búho, 2001.

Sepúlveda López de Mesa, Rodrigo Iván. “Vivir las ideas, idear la vida. Adversidad, suicidio y flexibilidad en el ethos de la Emberá y Wounaan de Riosucio, Chocó". Antípoda: Revista de Antropología y Arqueología, 6 (2008), pp. 245-269.

Tapia, Patricia I.; Vohringer, Paul y Ornstein, Claudia. “Conducta suicida en el hospital general". Revista Hospital Clinico Universidad de Chile, 21 (2010), pp. 187-196.

UNICEF (Ed). Suicidio adolescente en pueblos indígenas: tres estudios de caso. Panamá: UNICEF, 2012.

Adriana Maroto Vargas. Costarricense, obtuvo su maestría en Sociología por la Universidad de Costa Rica. Se desempeña como docente e investigadora en la Escuela de Psicología de la Universidad de Costa Rica. Forma parte de iniciativas ciudadanas feministas y de defensa de los derechos sexuales y reproductivos. Recientemente ha estado trabajando el tema de suicidio desde la acción social y la investigación en la Universidad anteriormente mencionada.

Contacto: adriana.maroto_v@ucr.ac.cr

ORCID: orcid.org/0000-0002-9899-9566

Carolina Castillo Echeverría. Costarricense, obtuvo su maestría en Ambiente, Desarrollo y Paz por la Universidad para la Paz y la Maestría en Liderazgo y Gerencia Ambiental por la Universidad para la Cooperación Internacional. Actualmente es 
candidata para obtener el título de doctorado en Sociología y Antropología Sociología en la Universidad Central Europea. Anteriormente se desempeñó como docente e investigadora en la Escuela de Sociología de la Universidad de Costa Rica donde trabajó el tema de suicidio desde la acción social y la investigación.

Contacto: carolina.castillo_e@ucr.ac.cr

ORCID: orcid.org/0000-0002-4792-0029 